\title{
2MASS Observations of the Large Magellanic Cloud
}

\author{
S.D. Van Dyk, R. Cutri \\ IPAC/Caltech, Pasadena, CA 91125, USA \\ M.D. Weinberg, S. Nikolaev, M.F. Skrutskie \\ Dept. of Physics \& Astronomy, Univ. of Massachusetts, Amherst, MA \\ 01003, USA
}

\begin{abstract}
The Two Micron All Sky Survey (2MASS) has observed a portion of the Large Magellanic Cloud (LMC) at $J(1.25 \mu \mathrm{m}), H(1.65$ $\mu \mathrm{m})$, and $K_{s}(2.17 \mu \mathrm{m})$, as part of its routine nightly operations with an automated $1.3-\mathrm{m}$ telescope at CTIO, Chile. The camera observes the sky in the three channels simultaneously, using $256 \times 256 \mathrm{HgCdTe}$ detector arrays. The survey samples the sky in $6^{\circ} \times 8.3$ scans. The 2MASS Production Processing System provides final atlas images and source extractions with precise photometric calibration and astrometric positions. The survey's $10 \sigma$ sensitivity is $15.8 \mathrm{mag}$ at $J, 15.1$ at $H$, and 14.3 at $K_{s}$. 2MASS will ultimately detect $\sim 10^{7}$ point sources in the LMC and will support analyses of the ages, luminosity and mass functions, and metallicities of the red stellar populations and a census of AGB and carbon stars, as well as extinction maps, across the LMC. This work presents an initial analysis of $\sim 20$ square degrees.
\end{abstract}

\section{Introduction}

The LMC was observed by 2 MASS as part of routine nightly southern operations in $1998 \mathrm{March}$ and April. These observations covered mostly the eastern half of the galaxy and required $\sim 4$ hours total observing time. Sample 2MASS Atlas Images of selected regions of the LMC can be found in the Image Gallery at http://www.ipac.caltech.edu/2mass/.

\section{Preliminary Results}

Production processing resulted in $\sim 10^{6}$ source extractions. In Figure 1 we show the color-color and color-magnitude diagrams, respectively. The observed dispersion is due to differences in ages, metallicity, and, predominantly, crowding. On each diagram we have overlaid isochrones from Bertelli et al. (1994) for the appropriate metallicities, assuming $m-M=18.5$ (Panagia et al. 1991).

The diagrams are dominated by intermediate-age $\mathrm{K}$ and $\mathrm{M}$ giants, with ages $\sim 2$ Gyr (for $Z=0.008$ ) or $\sim 5-6$ Gyr (for $Z=0.004$ ). In the inner galaxy young main sequence stars, and blue and red supergiants are apparent, with ages to 

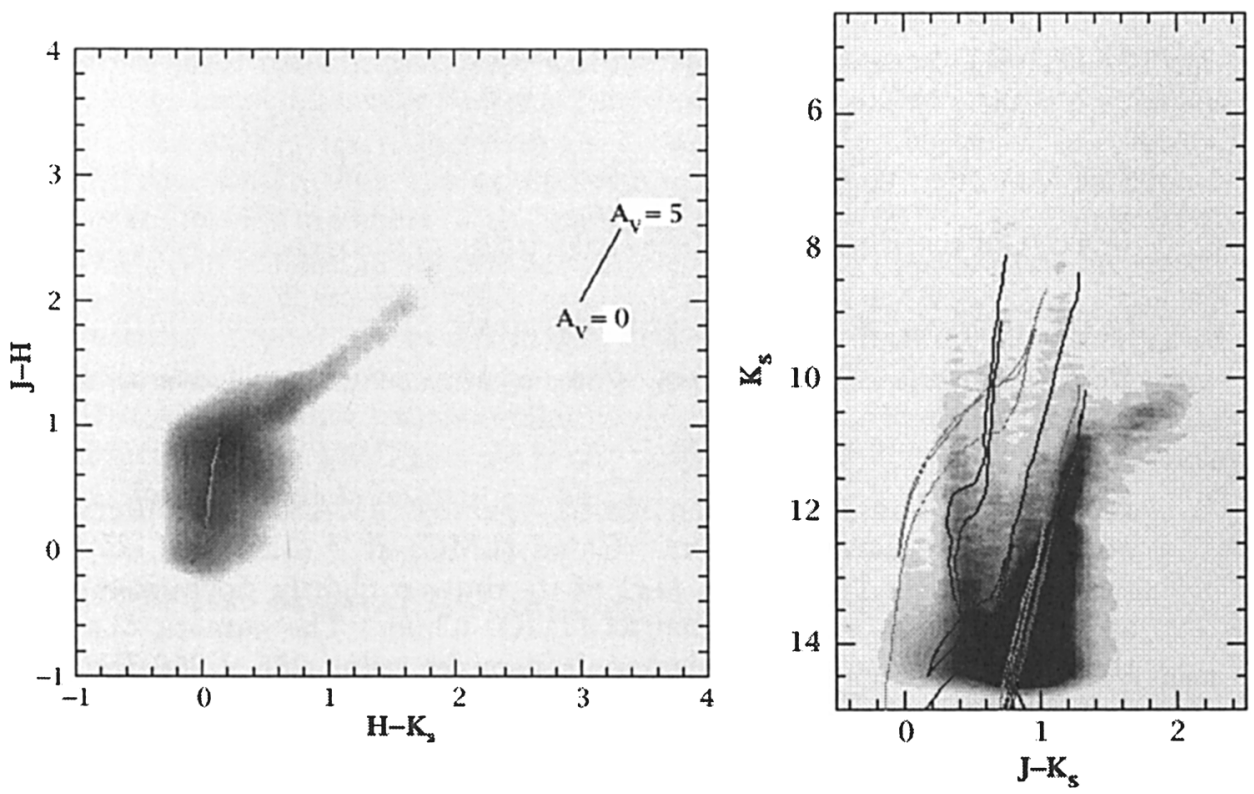

Figure 1. Color-color and color-magnitude (Hess) diagrams for the LMC, based on 2MASS source extractions. Isochrones have been overlaid, representing various LMC populations and foreground dwarfs and giants.

Z100 Myr. There are also bright AGB stars, carbon stars, and dust-enshrouded AGB stars; conservative estimates are $\sim 2500 \mathrm{C}$ stars and 550 obscured AGB stars. An ancient population, with $Z=0.001$, is not obvious from our diagrams.

The stellar halo of the LMC can be modeled to derive a lower limit on the LMC mass. We have selected twelve $0.5 \times 0.5$ subfields $2^{\circ}-5^{\circ}$ from the LMC center. We have applied the Maximum Likelihood method to parameterized models of the spatial density and luminosity function, assuming both a Gaussian and a power-law $(\gamma=2)$ density profile. Both density profile models give a tidal radius of $10.8 \mathrm{kpc}$ and $M_{\mathrm{LMC}} \gtrsim 1.0 \times 10^{10} M_{\odot}$, consistent with the star counts of the LMC giants. Additionally, we find an optical depth due to microlensing of $\tau \sim 1.7 \times 10^{-7}$. The fraction of the halo mass in the form of MACHOs is $\sim 0.25$.

\section{References}

Bertelli, G., et al. 1994, A\&A, 106, 275

Panagia, N., et al. 1991, ApJ, 380, L23 


\section{Discussion}

Hans Zinnecker: It is reassuring that ZMASS and DENIS find the same results! After all, science is not only about discovery, but also about independent confirmation.

Cecile Loup: DENIS has observed the whole central part of the LMC. In total about 50 square degrees. Data will get to the public in about one year. 